\title{
Let Your Robot Go - Challenges of a Decentralized Remote Robot Competition
}

\author{
Tim Laue ${ }^{1}$, Arne Moos ${ }^{2}$ and Patrick Göttsch ${ }^{3}$
}

\begin{abstract}
Holding an international robot competition, such as the RoboCup, usually requires many people and their robots to come together in one place for several days. In case of international travel restrictions, such an event cannot take place in its intended way. In this paper, we describe the German Open Replacement Event (GORE), a robot tournament for RoboCup Standard Platform League teams, which was organized in a decentralized way and required remote participation. Furthermore, we provide insights into the specific technical and scientific challenges the participating teams had to face, along with an outlook on implications for future robot competitions.
\end{abstract}

\section{INTRODUCTION}

For many years, various scientific robot competitions, such as the RoboCup or different DARPA Challenges, have been held all around the world. For these competitions, the researchers usually travel with their robots to defined competition sites, where they prepare, adapt, and configure their systems. In general, this also involves a dedicated setup phase of multiple days prior to the actual competition, which allows the researchers to fine-tune their robots. This phase is particularly important due to the competition environment, in which all robots are supposed to operate at their limits in terms of precision and speed. However, due to the coronavirus pandemic, all competitions of this kind have been cancelled since spring 2020.

This is why in spring 2021, the German Open Replacement Event (GORE) for the RoboCup Standard Platform League (SPL) was organized by the latter two authors. In contrast to other RoboCup World Cup alternatives (see Sec. II-C), this competition aimed for 5 vs. 5 robot football games with a standardized robot platform based on a rule set that only differed in details from the standard rules (see Sec. III-C). For this purpose, two competition sites were set up, to which all participating teams sent robots that were compiled to form two robot pools of 16 robots each. All humans stayed at home (except for a few people who handled the hardware on site) and were given remote access to a random subset of robots from the pool of a particular competition site for each game. The setup is a particularly challenging as there are certain trade-offs

\footnotetext{
${ }^{1}$ Tim Laue is with the Multi-Sensor Interactive Systems Group of the Department of Mathematics and Computer Science, University of Bremen, Germany t laueduni-bremen. de

${ }^{2}$ Arne Moos is with the Robotics Research Institute of the Department of Electrical Engineering and Information Technology, TU Dortmund University, Germany arne.moos atu-dortmund. de

${ }^{3}$ Patrick Göttsch is with the Institute of Control Systems, Hamburg University of Technology, Germany patrick.goettsch@tuhh. de

https://doi.org/10.15480/882.3718

978-1-6654-1213-1/21/\$31.00 @2021 IEEE
}

between a maximum robot performance and a certain kind of robustness given the partially uncontrollable hardware and environmental conditions. Furthermore, previously common manual calibration stages have to be omitted or to be carried out (semi-)automatically due to having only remote access to the robots and limited handling support from the arena's team assistant.

In summary, the two main contributions of this paper are the following:

- It describes the organization and technical infrastructure of a robot competition, which has successfully been conducted, that can be held remotely and decentralized.

- An overview is given of particular research and development challenges that participants face when competing with robots that are not on hand and not their own.

The remainder of this paper is organized as follows: In Sec. III. the RoboCup context of the GORE as well as related competitions are described. An overview of the organization, the technical infrastructure, and rule adjustments for the competition is given in Sec. III. followed by a description of the scientific challenges of the participants in Sec. IV. The implications of the competition are discussed based on the feedback of the teams in Sec. $\mathrm{V}$ and finally, the paper concludes in Sec. VI.

\section{BACKGROUND AND RELATED WORK}

This section describes the robot platform and the general setup of RoboCup SPL games. Furthermore, an overview and comparison to similar remote competitions is given.

\section{A. The RoboCup Standard Platform League}

In the wide field of artificial intelligence and autonomous mobile robotics, the RoboCup represents an important platform for research and connecting people. Research results are demonstrated in defined environments, and are exchanged on a subsequent symposium. The RoboCup SPL belongs to the RoboCup soccer section and focuses on playing soccer with autonomous small bipedal humanoid robots. The unique feature of this league is that all teams must use the same unmodified hardware from one manufacturer, but possibly in different versions. This puts a focus on the software as well as team play and allows, under normal circumstances, to perform competitive 5 vs. 5 games. A game is played in natural lighting on a 9x6 meter artificial turf field, organized like a human soccer field. The RoboCup SPL rule book [1] for competitions in 2020 has been adapted for GORE as outlined in Sec. III-C 


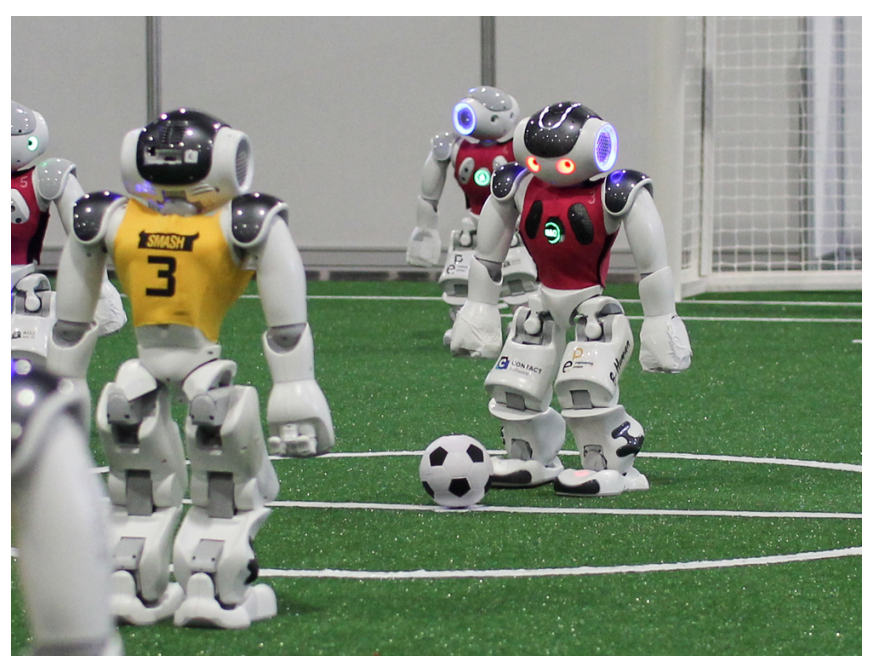

Fig. 1. NAO robots in a RoboCup SPL game.

\section{B. The NAO Robot Platform}

In RoboCup SPL, the NAO robot from Softbank Robotics 1 is used for now more than ten years, currently in its versions five and six. Some NAO robots in a SPL game are depicted in Fig. 1,

In the latest version six, the robot has a height of $57 \mathrm{~cm}$, a weight of $5.5 \mathrm{~kg}$ and 25 actuated joints. Two OV5640 cameras in the head, one below the other (non-stereo), are used to perceive and react to the environment. An Intel Atom E3845 quad-core processor is used together with four gigabytes of RAM to perform calculations. However, since no dedicated graphics card is available, only the lightweight, integrated one can be utilized. For communication purposes, a Gigabit Ethernet port and an IEEE $802.11 \mathrm{a} / \mathrm{b} / \mathrm{g} / \mathrm{n} \mathrm{WiFi}$ card are available. Furthermore, the robot is equipped with several sensors like 12 bit hall-effect sensors to measure each of the joint positions, a six degree-of-freedom inertial measurement unit (IMU), four ground contact force sensors for each foot, one foot bumper in each foot's tip, two sonars and four omnidirectional microphones.

Each NAO is delivered with a custom Linux operating system. RoboCup teams configure this operating system for their purposes and install their own software.

\section{Other Competitions}

In 2020, nearly all on-site events got cancelled for all leagues at RoboCup and regional competitions like the GermanOpen. During the days RoboCup 2020 would have been held, the different leagues offered virtual meetings. Some RoboCup leagues organized workshops, talks or some simulation competitions. During the remainder of the year 2020 and first half of 2021, more events have been held online like Virtual RoboCup Asia-Pacific 2020, RoboCup Japan Open 2020 and RoboCup Portugal Open 2021, which offered online events for the simulation leagues of the RoboCup sections soccer, work and home.

1 https://www.softbankrobotics.com/emea/en/nao
Most leagues that play robot soccer on different hardware have opted for simulator-based competitions for RoboCup 2021 with optional local components. For example, the RoboCup Humanoid League as well as the RoboCup SmallSize League switched to virtual games in simulators to have competitive games during RoboCup 2021.

Teams of the RoboCup SPL also switched their annual onsite events to virtual ones, like the virtual Robotic Hamburg Open Workshop (vRoHOW) ${ }^{2}$ in December 2020 for SPL and Humanoid League and the Robotic Dortmund Event Open (RoDEO) ${ }^{3}$ in March 2021 for SPL teams. The latter event already featured a common robot pool to take the first steps towards a decentralized event like the GORE.

For RoboCup 2021 the RoboCup SPL switched to local technical challenges, to be executed in teams' laboratories and judged by remote referees, as well as small competitive challenges, in other participating teams' laboratories, requiring only a few operating robots.

Outside the RoboCup context, multiple other remote competition formats exist, too. In [2], a framework for remote field robotics competitions is presented. These competitions take place in a simulated environment. A small competition, using a single real gripper robot, is described by [3]. The VEX Robotics Competition [4] claims to be the world's largest remote robot competition. However, it does not have any scientific approach and the robots are operated remotely, most of the time. [5], [6] focuses as well on students to get them interested in robotics. It will also be held online in 2021.

\section{GORE - The German Open RePlacement Event}

Due to the limited possibilities to play 5 vs. 5 robot soccer games in the SPL since spring 2020, as well as due to the decision to have no competitive soccer games during RoboCup 2021 and some successful first remote deployment test during vRoHOW 2020 and RoDEO 2021, the idea of the GORE was born in order to conduct some competitive remote games. The main goal of this event was to play friendly games, and not primarily ranked games, to evaluate this way of performing matches and to lower the probability of hardware failures.

This section briefly describes the organization, the technical infrastructure and necessary rule adjustments.

\section{A. Organization}

The characteristic of this league allowed that only the robots travel to some dedicated competition sites, called arenas, and all humans (except for a few people who actually handle the robots on site) stay at home.

With the specification of a maximum of eight participating teams, the average even number of participants of the GermanOpen events throughout the last years, there was also the need for two arenas, to limit the workload of handling games and robots in each arena, in which the games could then be played. A referee team for SPL games consists of a Head

https://rohow.de/2020/en/ https://naodevils.de/rodeo 
Referee, a Game Controller, and two Assistant Referees. So it was also a prerequisite that at least four people were allowed to enter an arena at the same time to set up robots and referee a match. Two arenas, in Dortmund and in Bremen (see Fig. 2), which provided a full-size field were selected to host games.

Despite the fact that the two arenas have the same field dimensions, artificial turf, line layout, and natural lighting from outside due to windows on at least two edges of the field, there are differences in the two arenas that the participating teams had to deal with. The artificial turf in Dortmund's arena has been in use for several years and is therefore somewhat more worn than the one in Bremen's arena, which is only used in special cases. Because of the different ceiling heights in the two arenas, it is difficult for the arena in Bremen to achieve artificial lighting via ceiling lights, especially in poor lighting conditions from outside. In addition, the environment outside the field is different for both arenas.

Since each participating team must own at least five robots to participate in RoboCup SPL, sending only four robots to one of the two arenas also allowed that each team could keep at least one robot at home for local testing. In total, this results in a robot pool of 16 robots for each arena, from which then ten robots were randomly selected for each game. Unfortunately, the manufacturer was not present for direct repairs of the robots, like in previous GermanOpen and RoboCup competitions, so these robot pools incorporate a backup to allow a dropout of up to six robots each due to hardware failures.

The event has been composed of one setup day and three competition days. For each game execution, a time-slot of three hours has been planned, consisting of:

- Setup-phase (one hour): Selection of robots and jerseys; flashing the robots with a pre-configured image consisting of the teams' software.

- Calibration-phase (one hour): Each team gets half an hour on the empty field to calibrate their robots, either fully automated or by accessing the robots from remote, supported by their responsible local assistant.

- Game-phase (one hour): During the game, the assistant referees had two tasks: They were members of the referee team, and they were also the local representatives of the team communicating with the head referee.

Taking these time-slots into account, the decision was made that on average no more than two games should be played per day and arena. This resulted in six games per arena for the three competition days, i. e. altogether twelve games.

Originally, the schedule of the GORE tournament was planned with two groups playing a group phase in one of the arenas and two finals each played one half in each arena. After a meeting with the participating teams, there was a switch to a Swiss System Tournament (see [7]), adopted by the organizer's, over four rounds to maximize the number of games per team, to find opponents of approximately equal strength, and to allow all teams to play in both arenas.
For communication with the teams, different tools have been used. Before the event, communication via e-mail had been the primary communication channel to inform teams but also to get fast responses. During the event, there was a switch to website and Discord. The latter one provides text and videos channels, so that people can easily write and talk to each other and, for instance, share their screen. While the website contained information, links, schedule, and game statuses for online visitors of the GORE. Discord was used to have regular meetings with the teams, like opening and closing ceremony and team leader meetings, as well as private communication channels for each team. Each arena had channels for the home and away team to allow communication with the team responsible assistant during setup, calibration, and game phases.

To reduce the effort of the hosting teams in supporting setting up and calibrating robots as well as during the games, rules have been extended to standardize the whole procedure (see Sec. III-C) and limit hardware failures. This required some effort from the teams to adjust their workflow to the new rules.

\section{B. Technical Infrastructure}

Given the fact that robot soccer games were played remotely for the first time, there were some technical challenges that needed to be addressed.

First, the teams required remote access to upload their software to their assigned robots and check their status. For this purpose, OpenVPN servers were set up in both arenas, which allowed the teams to connect to the same subnet where the robots were hosted. To reduce the effort in accessing them, they were assigned with standard IP addresses, changed afterwards by the team, from a DHCP server based on the robots' MAC addresses.

However, SSH access does not provide a global view of what the robot is actually doing on the field. Therefore, in both arenas, a camera setup with a wide angle camera was used to stream live to YouTubs ${ }^{4}$. This offered the advantage that the teams could see what their robots are doing in the setup and calibration phase, and to allow spectators to watch the games. In addition to this, important referee decisions were displayed as an overlay to the camera image, such as goals, the game phase, free kicks or penalties for individual robots.

It is important to record log data in order to be able to analyse what happened more precisely afterwards, small USB sticks were inserted into the heads of the robots.

\section{Rule Adjustments}

Given that each team obtains random robots from other teams, the issues of robot safety, strong wearing out, and avoiding of hardware damages needed to be addressed. In addition, a common setup had to be created so that the steps to be taken before the robots can play, such as setup, calibration or positioning of the robots at the start of the

4 https://www youtube.com/playlist?list= PLgXoR1zR6QKjZyA1viyaVZ7fqBji8EnPf 

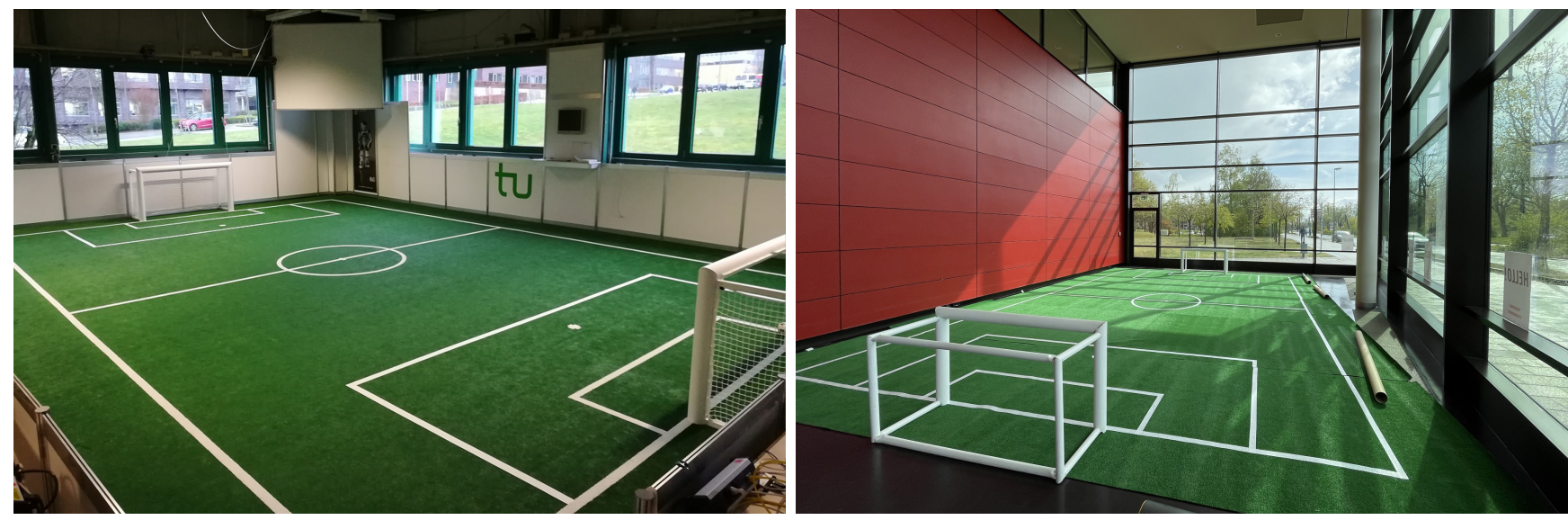

Fig. 2. The two GORE competition fields in Dortmund (left) and Bremen (right).

game, are streamlined as much as possible to enable the two hosting arenas to handle the robots easily and fast. For this purpose, the 2020 rule book was adapted (see [8]).

In order to guarantee a safe operation of the robots and to interrupt all actions of a robot in case of emergency, an implementation of a global safety gesture was forced, which makes it possible that the robot switches off its joints and does nothing more for the time being. Furthermore, in the robot's button interface there was an additional state for calibration, allowing for an automatic calibration of the robot to be started, which then only needs to be surveyed by the volunteers at the field.

To prevent unnecessary hardware failures and to move teams towards more careful handling of random robots, several rules were introduced to enforce this:

- The goalkeeper is not allowed (except for penalty kick) to dive, because this movement can be very destructive for the robot. Only the more gentle movement of a wide stance is allowed.

- In general, there is already a rule that you are not allowed to push/foul the opponent robots. However, this rule has been extended so that pushing within the own team leads also to this penalty.

- All teams have to deal with arbitrary robots in different states of wear. Therefore, it was introduced that each robot has only a maximum of two hardware related penalties in the first half, which then increases by one for the second half. These penalties apply to robots that stop moving, cannot stand up, or fall constantly, but also if a team issues a "request for pickup", for instance to restart a robot. If the limit is exceeded, this robot is ejected for the remainder of the game to not cause any hardware damages on a foreign robot due to potentially faulty software. In normal SPL games the usage of a sixth replacement robot is allowed, however for GORE it was not allowed.

- The head referee is allowed to perform a forced "request for pickup" whenever the robot hardware is in danger, which then also counts as a hardware related penalty for the offending team.

\begin{tabular}{ll} 
Team Name & Affiliation \\
\hline B-Human & Universität Bremen and DFKI \\
Bembelbots & Goethe-Universität Frankfurt am Main \\
BerlinUnited & Humboldt-Universität zu Berlin \\
HTWK Robots & HTWK Leipzig \\
HULKs & Hamburg University of Technology \\
NaoDevils & TU Dortmund \\
R-ZWEI KICKERS & Hochschule Kaiserslautern \\
\hline & \multicolumn{2}{c}{ TABLE I }
\end{tabular}

THE PARTICIPATING TEAMS OF THE GORE

- Overall, all referees were instructed to catch falling robots, if possible, in order to avoid more severe hardware damage to the robots. In normal games, this type of human intervention is not allowed.

\section{PARTICIPATION}

This section provides an overview of the participating teams along with their view of the particular scientific challenges of the competition.

\section{A. Participating Teams}

Seven teams participated in the GORE, see Tab. I. Except for one newcomer team, all of them have regularly attended RoboCup competitions for at least seven years and thus have developed working solutions for all problems of the domain in general. It stands out that all teams are based in Germany. This was not a formal requirement. The call for participation was distributed internationally, and there would have been a possibility to participate even without contributing to the robot pool.

Right after the end of the event, all teams were asked to participate in a short survey to provide feedback regarding their assessment of the event, their status, and their opinions about possible future events. All seven teams answered the survey and were informed about the possible publication of the results. 5

\footnotetext{
${ }^{5}$ Please note that the three authors are also members of participating teams. For the creation of the survey, other team members were not involved and vice versa, the authors were not involved in filling out the survey.
} 


\section{B. Preparation}

As there has never been any official competition with a similar setup, one can expect problems regarding the technical setup, as described in Sec. III-B This is why the survey asked for the team's preparation for the two major challenges, remote participation and using robots of other teams:

"Before GORE, did you ever test your software on your robots (not necessarily a full team) in a similar remote setup?"

"Yes (and it worked at least somehow)": 7/7

"Before GORE, did you ever test your software on the robots of another team (not necessarily a full team) in a similar remote setup?"

"Yes (and it worked at least somehow)": 5/7, "No": 2/7

There have been two more alternative answers: "We tried, but it did not work due to network-related issues." and "We tried, but it did not work due to problems in our software.". Both have not been selected by any team. This means, that all teams were prepared for participating in a remote competition and most teams were also able to deploy and run their software remotely on robots that are not their own. This provided a good base for the actual GORE competition, as major barriers were removed in advance. As described in Sec. III, it has to be mentioned that first at vRoHOW 2020 with a few teams and second at RoDEO 2021 with a larger number of teams, remote deployment with a similar setup has been tested. This was already used by most, but not all, teams for testing their systems. Despite limited lab access for some teams, all teams managed it to get their code and tools ready for remote deployment.

\section{Scientific and Technical Challenges}

As aforementioned, no major technical problem occurred during the competition. However, as mentioned in the introduction, the overall setup is challenging as sophisticated tuning for a competition usually requires the presence of the team at the actual competition site. Thus, the teams were asked about their opinion about major challenges in free text:

"Given the specific setup of the GORE competi-

tion, which aspects of your robot software do you

consider to be challenged most, in general?"

The following topics were mentioned most often by the seven teams (mentioning multiple topics was possible):

\begin{tabular}{ll}
\hline Motion (get up, walk, calibrate walk) & 5 \\
Camera Calibration & 4 \\
\hline
\end{tabular}

Other topics that were each mentioned once: Remote debugging, remotely detecting hardware issues and ball detection in extreme lighting conditions. Overall, these results clearly show that working on previously unknown robots of the same type on unknown, untestable ground appear to be the major issues.

Given these assessments, the teams had to answer this follow-up free text question:

"On which of these aspects did you actually work in preparation for the GORE competition?"

\begin{tabular}{ll}
\hline Motion (get up, walk, balancing) & 3 \\
Camera Calibration & 3 \\
Debugging, Infrastructure & 3 \\
None & 2 \\
\hline
\end{tabular}

In addition, the following topics were mentioned: calibration-free vision, self-localization and team play. This shows that many teams explicitly worked on the crucial issues for this particular competition. However, some admitted that they did not prepare for things they considered as important.

Independently of this competition, the RoboCup SPL continuously pushes towards more challenging conditions under which games are held. Among other things, varying lighting conditions, such as those at the two GORE fields (see Fig. 2), are now a commonplace at most other competitions. Thus, we asked the teams:

"In previous years, did you work scientifically on topics that turned out to be particularly useful for GORE? If there are any related publications of your team, please feel free to name them."

Answers were again accepted as free text. The following topics were mentioned most often by the teams (mentioning multiple topics was possible):

\begin{tabular}{ll} 
Calibration-free Robot Vision & 6 \\
Framework / Infrastructure & 3 \\
Motion (balancing, getting up, robust walk) & 3 \\
None & 2 \\
Camera calibration & 1 \\
\hline
\end{tabular}

The answers mentioned multiple finished theses about different topics. For the dominating topic, Calibration-free Robot Vision, multiple research publications were referenced. Due to the aforementioned trend towards natural lighting, a major shift from classic color-class-based image segmentation techniques towards Deep Learning approaches can be observed throughout the previous years. This has also been reinforced by the increase of processing power in the NAO V6 model. An early work that describes a lightingindependent approach for robot vision in the SPL context was published by [10]. Implementations for efficient neural network inference on the NAO platform have been provided by [11] as well as by [12]. The classification of preprocessed image patches, e.g. for ball classification, has become the de facto standard, one example out of many is given in [13]. A Deep Learning approach for processing the whole image for the purpose of robot detection has been presented by [14]. To be able to provide pre-configured images, in [15], code and instructions were published to set up custom images. This can also be a base for autonomous setup and calibration.

\section{Results}

Overall, the GORE can be considered as a successful event. At the two arenas, 12 games were held. Nearly all games took place within their scheduled time slots and almost all teams were able to let their software run in each of these games. Most issues were solved after the first competition day. In addition, the rule adjustments regarding 
the protection of the robot hardware proved effective and led to a total of only 3 ejected robots across all games. This is considered very positive, since the teams could only calibrate their robots remotely.

As the GORE was both, a competition as well as a new experimental environment that allows teams to test their robots in a new challenging environment, not only the mere results but also the teams' view on the competition is an important aspect. Thus, in the survey, the teams were asked the following two questions, both with a linear answering scale from 1 to 10 (higher is more positive):

\begin{abstract}
"After GORE, do you think that your team is able to cope with most challenges involved in operating your software on robots of another team in a remote setup?"
\end{abstract}

Result: $\varnothing$ : $9.43 \quad$ Range: 8 - 10

“Compared to your team's performance in "normal" games, how did your software work during GORE?"

\section{Result: $\varnothing$ : $7.83 \quad$ Range: 6 - 10}

This proves that the proposed setup is practical for the participating teams. However, in average, the teams see a clear decrease in game performance. Only one team did not notice any difference, whilst two teams considered their performance as almost halved ${ }^{6}$ When asked about their biggest issues while participating in the GORE competition, multiple teams mentioned the limited access to robots as well as minor hardware defects in the robots they had been assigned to. Furthermore, one of the two teams running an arena stressed the fact that participating and organizing at the same time is a major issue, if the team is too small.

Although the GORE was not an official tournament, a final competition result was determined. Like in normal RoboCup football events, the scoring was based solely on the goals the teams achieved in a match, without any extra points awarding specific remote capabilities. All detailed results can be found at the competition website [9].

Compared to the goals scored in an ordinary competition, the GORE also seems to be a quite realistic alternative. At the GORE, the participating teams scored 75 goals in 12 games, resulting in an average of 6.25 goals per game. This can be compared to the round of last eight of the last RoboCup world cup in 2019, which provided the most recent RoboCup results. Furthermore, among these eight teams, five participants of the GORE were present. In the seven final games of RoboCup 2019, 55 goals were scored, resulting in an average of 7.86 goals per game. This is slightly higher than at the GORE but not significantly different. The decrease of goals per game roughly resembles the teams' self-assessment of their decrease in performance. However, some chance could have been involved, as other factors (see Sec. III probably influenced the outcome of games at the GORE: goalkeepers were not allowed to dive, there were no replacement robots, and multiple penalties were handled in a stricter way.

${ }^{6}$ The newcomer team did not respond to this question

\section{LESSONS LEARNED AND IMPACT FOR FUTURE COMPETITIONS}

As described in the previous section, the event was successfully held and the participating teams were able to cope with the proposed scenario, performing not much worse than in a normal competition. However, one major questions remains to be answered: What do the insights and experiences gained at the GORE mean for future robot competitions? Again, the teams were asked multiple questions about their gain of knowledge and potential future competitions in a similar style. The first question again had a linear answering scale from 1 to 10 (higher is more positive):

"For a (post-pandemic) future, how useful do you consider your team's ability to carry out experiments remotely in a different lab?"

\section{Result: $\varnothing$ : $8.86 \quad$ Range: 8 - 10}

This can be considered as a very significant outcome of the GORE, possibly contributing to future scientific results. All teams are very convinced of having a new option for experiments. When looking forward to future robot competitions, this also becomes clear:

"Assuming that there will be "normal" competitions again, i. e. persons are on site and own robots are used, are there any aspects from GORE that you think will help you in future competitions?"

$$
\text { Result: } \varnothing \text { : } 8.86 \quad \text { Range: } 7 \text { - } 10
$$

In an additional field for free text, teams were asked to enter the topics they assumed to be of benefit for future competitions. Two topics dominated this field, as both were mentioned by a majority of teams:

\begin{tabular}{ll} 
Robot Setup and Code Deployment & 5 \\
Calibration & 4 \\
\hline
\end{tabular}

This clearly shows a trend towards less human intervention and more automation, which we consider as a significantly positive effect of the GORE. At future competitions, teams could save time and the organizers could be more flexible regarding the setup of the competition.

Regarding future options, the teams were asked three final questions, again with a linear answering scale from 1 to 10 (higher is more positive):

"Would you like to participate in an event like GORE in autumn this year."

\section{Result: $\varnothing$ : $9.86 \quad$ Range: 9 - 10}

"Would you like to participate in a league with one game per month in the time from October till May of the next year?"

Result: $\varnothing$ : $7.71 \quad$ Range: 1 - 10

"How beneficial do you think it would be to get (at least some) arbitrary robots from a robot pool also in future RoboCups (in presence)?"

Result: $\varnothing$ : 6.29 Range: 2 - 10

The teams obviously seem to want another event like the GORE. The alternative of playing some kind of a league is also wanted by most teams. The lower average score mainly 
results from one outlier by a team that gave the feedback that such a league would not fit its internal schedule. Finally, the teams seem to have a mixed view on a possible combination of the concepts of the GORE and a normal competition, i.e. having a randomized robot pool at future RoboCups. The answers were spread over almost the whole linear range, without significant clusters. We do not have any text feedback about this topic.

\section{CONCLUSIONS AND FUTURE WORK}

This paper described the organization and technical difficulties, as well as necessary rule adjustments that made a successful decentralized remote robot event, the GORE, possible. Despite the restrictions imposed by the global coronavirus pandemic, it was possible for the seven participating teams to play full 5 vs. 5 robot soccer games with NAO robots, not necessarily their own robots, on different fields. To facilitate full games, the participating teams had to make some adjustments to their robot software to remotely set up and then (automatically) calibrate their robots in a short time.

In the subsequent post-event survey, most teams indicated that they had prepared for the new challenges and had coped with them during the event, sometimes at the expense of maximum performance. The survey results also point out the fields of research that most teams considered to be important to cope with scenarios which do not allow full control over an own robot and the environment.

Reviewing this event, we can conclude that most of the ideas worked out, teams could set up unknown robots remotely and calibrate them in a short amount of time (semi)automatically. The predefined, streamlined user interface to safely handle robots turned out to be positive and should be retained, although unfortunately not all teams have complied with it exactly. The VPN connections had no problems during this event, and the livestream delay was only 24 seconds, but the technical infrastructure must be better evaluated in terms of capacity, performance and latency. One major point to investigate is the need for an evaluation criterion to find out the wear level of the robots. This would help to ensure that teams get equally worn out robots and provide a standard criterion when robots should be discarded. Especially the question if a robot is still fully functional has caused discussions.

For the future, one can expect to see more competitions like the GORE. As described, most teams appear to have a strong preference towards such events or other test games. Independently of any future pandemic circumstances, the developed infrastructure for remote operation and for deploying the own software to other robots will provide additional inexpensive experimental opportunities without the need to travel. All pre-configured software has been uploaded, is available for the teams and can be configured utilizing local configuration files. Another big step into the future is, that by utilizing remote access to perform games, the SPL community gets much more connected over the year than on one or two single events. Thus, teams can perform test games against other teams' code in their absence in the local arena. Furthermore, the RoboCup 2021, which partially used a similar setup, already benefited of these new remote capabilities. So, remote games can support the regular RoboCup competitions over the year.

\section{ACKNOWLEDGMENTS}

The authors would like to thank all participating teams for making this event possible and for taking part in our survey. Special thanks go to the members of the NaoDevils and the B-Human team, who operated the two competition sites despite the difficult pandemic situation.

\section{REFERENCES}

[1] RoboCup Technical Committee, "RoboCup Standard Platform League (NAO) Rule Book," 2020, only available online: https://collaborating. tuhh.de/HULKs/robocup_tc_public/raw/master/SPL-Rules_2020.pdf

[2] G. Cabrita, R. Madhavan, and L. Marques, "A framework for remote field robotics competitions," in 2015 IEEE International Conference on Autonomous Robot Systems and Competitions. IEEE, 2015, pp. 192-197.

[3] N. Funk, C. Schaff, R. Madan, T. Yoneda, J. U. De Jesus, J. Watson, E. K. Gordon, F. Widmaier, S. Bauer, S. S. Srinivasa, et al., "Benchmarking structured policies and policy optimization for real-world dexterous object manipulation," arXiv preprint arXiv:2105.02087, 2021.

[4] The REC Foundation, "VEX robotics competition," 2021, only available online: https://www.roboticseducation.org/vex-robotics-competition/

[5] First, "A GLOBAL ROBOTICS COMMUNITY PREPARING YOUNG PEOPLE FOR THE FUTURE," 2021, only available online: https:// www.firstinspires.org/

[6] Best Robotics Inc., "Inspiring students to pursue careers in engineering, science, technology, and mathematics through robotics design..." 2021, only available online: https://www.bestrobotics.org/site/ documents/formats/BEST_Online_Competition.pdf

[7] Wikipedia, "Swiss-system tournament - Wikipedia, the free encyclopedia," http://en.wikipedia.org/w/index.php?title=Swiss-system\% 20tournament\&oldid=1022448614 2021, [Online; accessed 15-May2021].

[8] RoboCup Technical Committee, "GORE Standard Platform League (NAO) Rule Book," 2021, only available online: https://collaborating. tuhh.de/HULKs/gore/-/raw/master/GORe-Rules.pdf

[9] A. Moos and P. Göttsch, "GORE 2021," https://gore2021.netlify.app 2021, [Online; accessed 15-May-2021].

\section{SURVEY REFERENCES}

[10] I. Schwarz, M. Hofmann, O. Urbann, and S. Tasse, "A robust and calibration-free vision system for humanoid soccer robots," in RoboCup 2015: Robot World Cup XIX. Springer, 2015, pp. 239-250.

[11] F. Thielke and A. Hasselbring, "A JIT compiler for neural network inference," in RoboCup 2019: Robot World Cup XXIII, ser. Lecture Notes in Artificial Intelligence, vol. 11531. Springer, 2019, pp. 448456.

[12] O. Urbann, S. Camphausen, A. Moos, I. Schwarz, S. Kerner, and M. Otten, "A c code generator for fast inference and simple deployment of convolutional neural networks on resource constrained systems," 2020.

[13] T. Röfer, T. Laue, G. Felsch, A. Hasselbring, T. Haß, J. Oppermann, P. Reichenberg, and N. Schrader, "B-Human 2019 - complex team play under natural lighting conditions," in Robot World Cup, ser. Lecture Notes in Artificial Intelligence, vol. 11531. Springer, 2019, pp. $646-657$.

[14] B. Poppinga and T. Laue, "JET-Net: Real-time object detection for mobile robots," in RoboCup 2019: Robot World Cup XXIII, ser. Lecture Notes in Artificial Intelligence, vol. 11531. Springer, 2019, pp. 227-240.

[15] HULKs, "meta-nao - yocto layer for softbank nao v6," https://github. com/HULKs/meta-nao. 2021, [Online; accessed 16-May-2021]. 